\section{Pathophysiology Haemostasis and Thrombosis}

\title{
Computer Assisted Anticoagulant Therapy
}

\author{
C. Manotti, C. Pattacini, R. Quintavalla, A. Tagliaferri, M. Lombardi, \\ M. Tassoni
}

Centro per le Malattie dell'Emostasi, Azienda Ospedaliera di Parma, Italy

\section{Key Words \\ Anticoagulant treatment - Computer decision support sistems · Management decentralisation · General practitioners}

Thrombosis Centre in terms of time spent by patient in therapeutic range.

Copyright @ 2004 S. Karger AG, Basel

\section{Introduction}

It has been demonstrated that there is approximately an exponential relationship between TTR and major episodes of bleeding. Small departures from the target range were associated with small-to moderate increases in bleeding rates, while large departures were associated with large increase in bleeding rates. A similar relationship was found between TTR and thromboembolic events. Anticoagulant treatment needs to be monitored and adjusted to prevent thromboembolic events avoiding haemorrhage [1-6].

The constantly workload increase has led to the development of computerised decision support systems for a better management of patient care. Many computerised programs have been developed and introduced in clinical practice first in the Netherlands at the Thrombosis Service in Leiden since 1972 by means of "BASAK AND DOSAK" program [7] and later Italy since 1986 by means of the "P.A.R.M.A. (Program for Archiving Refertation and Monitoring Anticoagulants)" system [8] and also in UK by DAWN program [9]. PARMA system is now widely used by several anticoagulant clinics in Italy on the basis of good results observed in local experience.

\section{KARGER}

Fax +41613061234

E-Mail: karger@karger.ch

www.karger.com
(C) 2004 S. Karger AG, Basel

1424-8832/04/0336-0366\$21.0/0

Accessible online at: www.krager.com/pht
CesareManotti, MD

Centro per le Malattie dell'Emostasi

Azienda Ospedaliera di Parma

Via A.Gramsci 14,

I-43100 Parma, Italy

Tel: + 390521 702239; E-mail: cmanotti@ao.pr.it 


\section{Computerized Decision Support Systems}

Computerised decision support system (CDSS) can be defined as "programmes designed to give advice about significance of results and their implications for subsequent patient management" [10].

Many clinical situations have been investigated to verify the utility of CDSS: few have demonstrated stable effects. One area where success has been reported is the field of oral anticoagulation management.

To be useful, CDSS must provide a database of information about patients, clinical and follow-up data, which must be available on-screen for easy consultation during dosage prescription.

Most of these systems use two main modules:

- An induction module for suggesting the first maintenance dose following the priming dose based upon the individual biological response (INR at 4th day) to the drug loading dose (PARMA system) or by an INR daily algorithm set by every centre according to the local clinical practice (DAWN).

- The second module (maintenance module) proposes confirmation of previous dosage (stable patients) or suggests a dose variation on the basis of logical criteria derived from rules usually applied by physician in the dosage prescription and by a mathematical equation derived from regression slopes for variation of INR values on dose of anticoagulant for a range of weekly dosages.

The time of next test is also suggested using a set of variables comparing actual INR, previous interval between tests, previous INR in therapeutic range and dosage stability.

\section{Computerized Decision Support Systems and Effectiveness}

Many studies on computer decision support system have been done in last years [12-17]. All published studies demonstrated good results but all are carried out in single centre and most of them are not randomised and no one is multicentre study.

To verify the safety and efficacy of CDSS are necessary in fact multicentre studies to assess more reliable comparisons between doses generated by computer and those prescribed by medical staff.

Only two randomised multicenter studies have been published recently: one by Poller [18] in 1998 and another by an Italian group in 2001 (APROAT study [19]). In both study the quality of treatment was assessed with the method pro-

Computer Assisted Anticoagulant Therapy posed by Rosendaal [20], in which the percentage of time spent in therapeutic range was measured, defined as Time In Therapeutic Range (TTR).

In 1998 Poller published the first randomised prospective multicentre study on the CDSS in which he demonstrated the effectiveness of computer assisted dosage in improving the time spent into therapeutic range in comparison with traditional method.

For all patients combined (stabilisation and stable patients) computer generated dosage gives a better control in achieving INR target range (63,3\% in computer group vs. $53,2 \%$ days in range in traditional group; $p=0.004)$. When analysed separately the stabilised patients faired significantly better with computer dosage than with traditional, but benefit in stabilisation no reach the statistical significance. Poller concluded that CDSS gave a better INR control than experienced physician did but clinical out come (haemorrhage and thromboembolic events) remain to be assessed together with cost effectiveness

The aim of the APROAT study carried out with PARMA system was to investigate if in similar clinical and laboratory conditions the use of CDSS could contribute to the improvement of the quality of OAT. We enrolled and randomised, in five Italian Centres, 335 patients in induction phase 145 in computer generated dose-C, 190 in traditional management-M and 916 patients in stable phase 458 in computer generated dose-C, 458 in traditional managementM.

In conclusion we found that patient in $\mathrm{C}$ group reached a stable state significantly earlier (39\% vs. $27 \%$ in $1^{\circ}$ month - $\mathrm{p}<0.01$ ) and spent more time within therapeutic range $(71,2 \%$ Vs $68,7 \%$ - $\mathrm{p}<0.001)$ in comparison with $M$ patients The improvement in treatment quality was obtained by reducing the time spent by patients in $\mathrm{C}$ group under range A reduction of the number of patient/visit per years was also observed

But these results could potentially reduce adverse effects but this should be tested in a large specifically designed study

On the basis of randomised multicenter studies we can conclude that CDSS introduction may improve the time in therapeutic range and also the management of a Anticoagulant clinic.

To definitely assess the real benefit of CDSS in reducing the prevalence of clinical adverse events of OAT, large specifically designed trials are necessary in the framework of multicentre studies.

In 2003 an European project totally supported by the European Commission started. This investigation concerns 40 European centres which will be involved in the project co-ordinated by Prof. Poller.

Six-teen thousand patients will be enrolled to achieve

Pathophysiol Haemost Thromb 2003/2004;33:366-371 
statistically significant clinical end points. 8 thousand patients will be randomised to receive dosage through traditional method, while 8 thousand will be randomised in CDSS, 20 Centres will use DAWN program and the other 20 PARMA System

The first trial objective is to determine whether a significant clinical benefit results from CDSS or not. The degree of improvement by CDSS in achieving therapeutic target INR will also been assessed. Finally it will be investigated if CDSS may prevent bleeding and thromboembolic events that would have considerable cost-saving implications.

\section{Computerized Decision Support Systems and Costs Saving}

In our Centre the introduction of "Parma system" including the web link strategy which connects the different units of the thrombosis centre: reception, venous puncture room, laboratory and therapy prescription allowed a considerable reduction of work load and decrease of patients waiting time with more efficient organisational work flow. Consequently a major cut in the management expenses can be reached by improving the service efficiency.

In Figure 1it can be observed a comparison between costs management of traditional prescriptions and the computerised systems. Costs for a single visit have been quantified by adding the three different parameters involved: fixed, variable and general costs. Fixed costs, not dependent on the patient management system, including reagents, disposable and instruments pay off, must be considered equal for the two systems. Variable costs strictly depend on the patient management and are considered in relation to the salary of physicians, nurses and technicians involved. General costs depend on heating, cleaning, phone expenses etc. and are calculated on the basis of $20 \%$ of previous costs. In the traditional management prescription, clinical and laboratory data storage, work list and results transcription and dosage prescription report are manually executed on paper forms. Parma system allows informatic storage of patient data and prescription print out and carries out the global

$\begin{array}{ll}\text { Fixed Costs } & \text { Manual } \\ \text { Variable Costs (labor) } & 0.66 \longrightarrow 0.66 \\ \text { General Costs } & 7.75 \longrightarrow 6.54 \\ \text { TOTAL } & 1.68 \longrightarrow 6.24 \\ \text { Savings per patient } & \end{array}$

Fig. 1. Comparison between cost management of traditional prescription and computerised system (using P.A.R.M.A. system web strategy) informatization of all units of the thrombosis centre. On this basis we calculate for each visits a cost of ten euro for Manual and six euro for Parma system. It can be observed that the money saving is about $38 \%$ for each visit in relation to the management system involved. Therefore the money saving is well defined if the computerised management is introduced.

\section{Computerized Decision Suppot Systems and Decentralisation of Oral Anticoagulant Managing}

The number of patients receiving oral anticoagulant therapy is constantly increasing world-wide.

In Figure 2 it is reported the increase of workload of our clinic. We have an exponential increase of new patient from 181 to 1440 from 1993 to 2002.

This trend has been confirmed also by a survey of the Italian Federation of Anticoagulant Clinic (FCSA). In fact, we found among 72 centres participating to therapeutic quality control exercise in 2003 a mean increase of new patients of 29\% from 2002 to 2003.

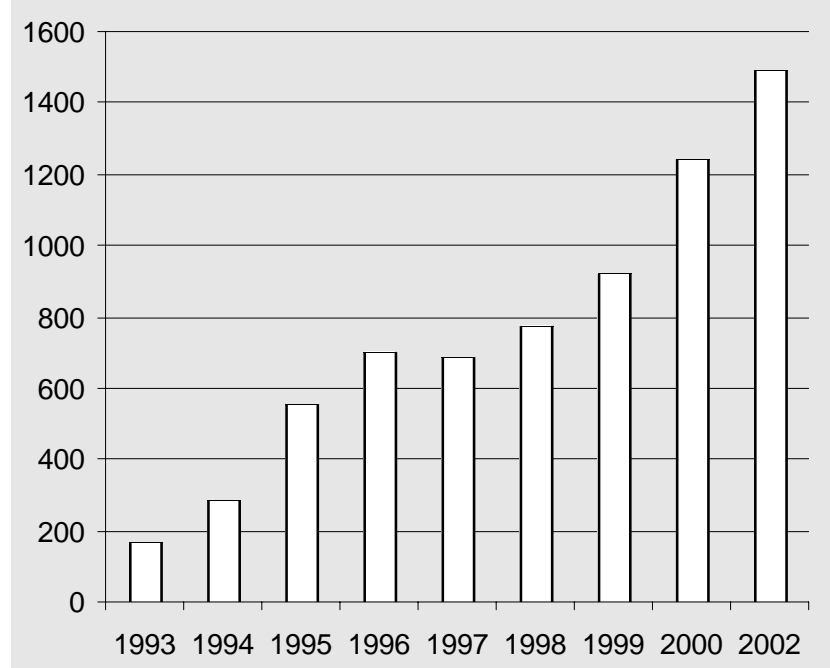

Fig. 2. Work load increase of Parma Thrombosis Centre (new patients enrolled)

Because of this increasing of workload for thrombosis service, alternative models have been developed:

- Primary cares with intervention of professional nurses

- Patients itself

- General practitioner 
Primary Cares with Intervention of Professional Nurses

In all these new models of delivery oral anticoagulant control CDSS will be used.

In a recent study Fitzmaurice [21] demonstrated that CDSS can reduce the reliance on specialist delivery of anticoagulant care, enabling other health care professional (nurses for example) to manage OAT. Use of near-patient testing offers the possibility of devolution of warfarin management in primary care.

Fitzmaurice concluded that oral anticoagulant care can be provided as well in primary care, through a nurse-led clinic, involving near-patient testing and CDSS, compared with routine hospital management

\section{Patients Self-Testing or Self-Management}

Patient self-testing or self-management may provide the greatest degree of decentralisation. Also in this model CDSS may be useful.

Patient analyses himself his own blood.

After self-testing he can refer directly to the Thrombosis Service. Expert physician of thrombosis service with the help CDSS send back the new dose.

In self-management the patient itself can obtain next dose and time of next test by a simplified CDSS implemented in near-patient testing device.

\section{General Practitioner}

General practice is another model for the delivery of anticoagulant control service, in which CDSS may maintain the efficacy and quality of therapy.

A similar model has been developed, using PARMA system in our city. We carried out a pilot study on the decentralisation of anticoagulant delivery in collaboration with some General practitioners (GP) of Parma area and now we are able to show the results of this new model of delivering OAT.

The aims of our study are :

- Give to GP the opportunity to directly monitor their own patients with the use of near-patient device and the informatic connection (via WEB) to central CDSS .

- Reduce the number of patient/visits at Thrombosis Center

- Improve relationship between GPs and their patients

- Improve patients quality of life

- Compare clinical and TTR outcomes.

In this study were involved 15 GP of Parma area. All GP before starting management of OAT in their own received a complete educational package including :

- Information on management

- Trial in Thrombosis Centre

- Education on the use of PARMA system

Then every GP received a password to connect themselves with central CDSS via internet explorer.

All GP patients participants to the study $(n=181)$ were managed by their own physician during 2003, for a total of 2234 visits (Figure 3 ).

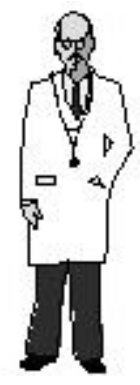

$$
\text { GP } n=15 \quad \text { GP training }
$$

Connection to central CDSS

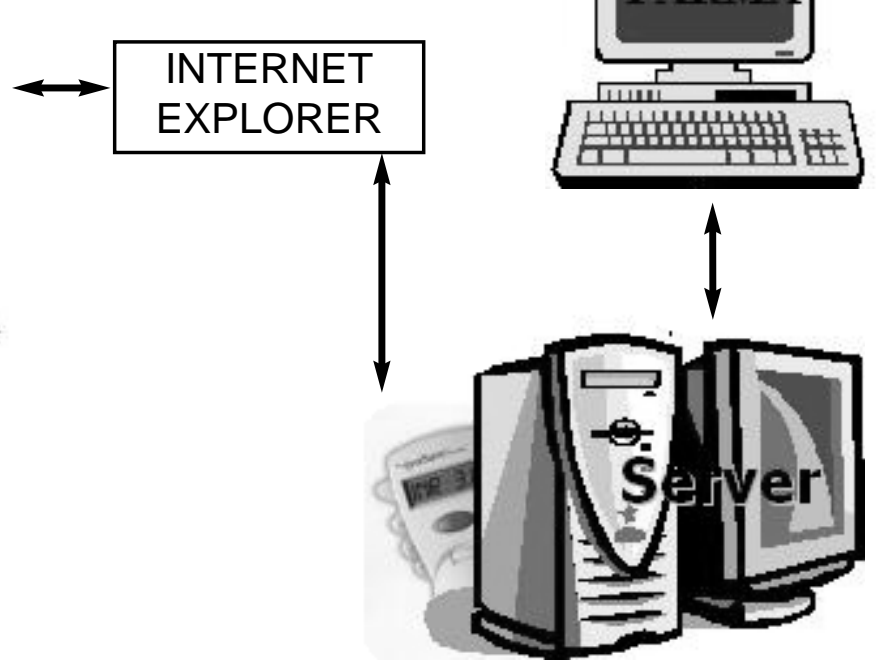

Fig 3. Work up scheme of GP alternative model of delivering anticoagulant monitoring 
The workup scheme GP alternative model to deliver OAT :

- Patients attend directly the general practice office

- Capillary blood is taken and the blood is analysed using near-patient testing device

- Dosing of anticoagulant is provided by general practitioner with the help of CDSS through a connection with central CDSS

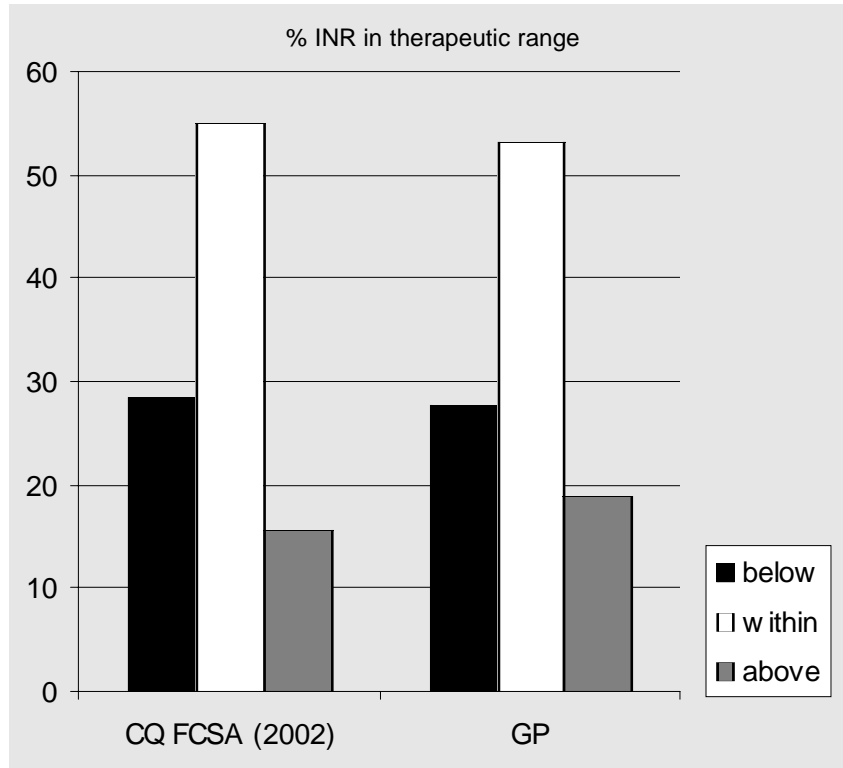

Fig. 4. Comparison of INR values obtained by GP and those of expert physician of Anticoagulant Clinics participating to 2002 therapeutic quality control of FCSA exercis

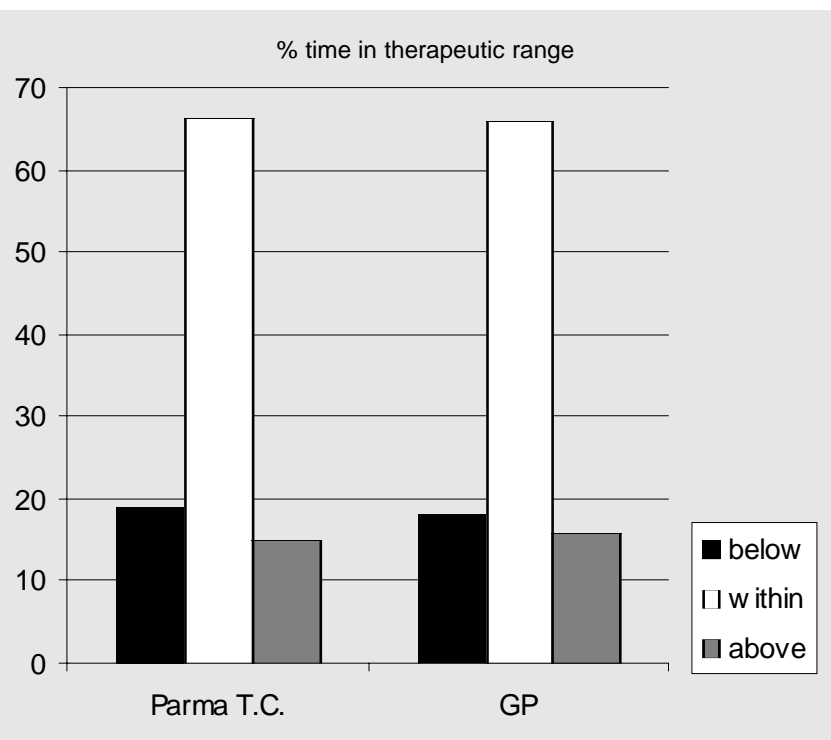

Fig. 5. Comparison of TTR in patient attending Parma Thrombosis Centre the year before the study and in the year in which OAT was delivered by their own GP
The new dosage is prescribed, printed and given directly to the waiting patient.

During the entire study period, GP delivered more than 2000 visits and 54\% of INR values were in therapeutic range. These results are very similar to those obtained by expert physicians of Thrombosis Centres participating to the 2002 survey of FCSA(Figure 4). In that case the mean INR in range were $56 \%$. An interesting improvement is present with respect to the days interval between visits: 15 vs 16 days respectively and also the number of visits per years carried out by GP are lesser than the ones of the Thrombosis Centre: 22 vs 25 respectively.

In Figure 5 is reported the comparison of TTR obtained in patients attending our clinic during the year before the study and in the year in which the OAT was delivered by their own Physician.

As we can observe a not significant difference between the two period in TTR emerged. In fact patients followed by GP have a TTR of $65.9 \%$ in comparison to Thrombosis Centre expert physician TTR 66.1\%

\section{Conclusion}

CDSS system has demonstrated to be able to improve the treatment quality in comparison to manual method, increasing the period of time spent by patients in computer group within the range.

Probably the use of CDSS may reduce adverse events.

It must be considered that implementation of web strategy allows an important financial saving as compared to the traditional management.

Improved success in achieving clinical benefit must be demonstrated by specifically designed studies.

We also must consider the future scenario for CDSS. In fact the constant increase of patients number and their pressure on thrombosis centres had led to the development of alternative models for delivery OAT: Primary care, General Practitioner, Patient self testing and self management. The use of CDSS has been central to the decentralisation process and may be useful in maintaining the efficacy and quality of anticoagulant control.

We demonstrated that GP are able to deliver OAT as well as expert physician of Thrombosis Centre in terms of time spent by patient in Therapeutic range.

We hope that all the opportunity offered by CDSS will let us and our patient to steer more safely in the more "quiet" sea of OAT. 


\section{References}

1 Petitti DB, Strom BL, Melmon KL. Duration of warfarin anticoagulant therapy and the probabilities of recurring thromboembolism and hemorrhage. Am J Med 1986;81:255-259.

2 Landefeld CS, Goldman L. Major bleeding in outpatients treated with warfarin: incidence and prediction by factors known at the start of outpatient therapy. Am J Med 1989;87:144-152.

3 Fihn SD, McDonnell M, Martin D, et al. Risk factors for complications of chronic anticoagulation: a multicentre study. Ann Intern Med 1993;118: 511-520.

4 Gitter MJ, Jaeger TM, Petterson TM, et al. Bleeding and thromboembolism during anticoagulant therapy: a population-based study in Rochester, Minnesota. Mayo Clin Proceed 1995;70:725-733.

5 Palareti G, Leali N, Coccheri S, et al, on behalf of Italian Study on Complications of Oral Anticoagulant Complications. Bleeding complications of oral anticoagulant treatment: an inceptioncohort, prospective, collaborative study (ISCOAT) Lancet 1996;348:423-428.

6 Palareti G, Manotti C, D'Angelo A, et al, on behalf of Italian Study on Complications of Oral Anticoagulant Complications. Thrombotic events during oral anticoagulant treatment: Results of the inception-cohort, prospective, collaborative ISCOAT study. Thromb Haemostas 1997;78: 1438-1443.
Wiegman H, Vossepoel AM. A computer program for long term anticoagulation control. Comput Programs Biomed 1977;7:71-84.

8 Manotti C, Pini M, Pattacini C, et al. Computer assisted prescription of oral anticoagulants. Thrombos Res 1991;64(Suppl 13): 192.

$\checkmark 9$ Poller L, Wright D, Rowlands M. Prospective comparative study of computer programs used for management of warfarin. J Clin Pathol 1993;46:299-303.

10 Delaney BC, Fitzmaurice DA, Riaz A, Hobbs FD. Can computerised decision support systems deliver improved quality in primary care? BMJ 1999;319:1281.

11 Hoffer EP, Marble KD, Yurchak PM, et al. A computer-based information system for managing patients on long-term oral anticoagulants. Comput Biomed Res 1975;8:573-579.

12 Routledge PA, Davies DM, Bell SM, et al. Predicting patient's warfarin requirements. Lancet 1977;ii:854-855.

13 Williams DB, Hart RC. A simple technique for predicting daily maintenance dose of warfarin. Am J Surg 1979;137:572-576.

14 Vadher B, Patterson DLH, Leaning M. Evaluation of a decision support system for initiation and control of oral anticoagulation in a randomised trial. Br Med J 1997;314:1252-1256.

15 Wilson R, James AH. Computer-assisted management of warfarin treatment. Br Med J 1984;289: 422-424.
16 Ryan PJ, Gilbert M, Rose PE. Computer control of anticoagulant dose for therapeutic management. Br Med J 1989;299:1207-1209.

17 Kubie A, James AH, Timms J, et al. Experience with a computer-assisted anticoagulant clinic. Clin Lab Haematol 1989;11:385-391.

18 Poller L, Shiach CR, MacCallum PK, et al, on behalf of European Concerted Action on Anticoagulation (ECAA). Multicentre randomised study of computerised anticoagulant dosage. Lancet 1998;352:1505-1509.

19 Manotti C, Moia M, Palareti G, Pengo V, Ria L, Dettori AG. Effect of computer-aided management on the quality of treatment in anticoagulated patients: a prospective, randomized, multicenter trial of APROAT (Automated PRogram for Oral Anticoagulant Treatment). Haematologica 2001;86:1060-1070.

20 Rosendaal FR, Cannegieter SC, van der Meer FJM, Briet E. A method to determine the optimal intensity of oral anticoagulant therapy. Thromb Haemostas, 1982;47:230-231.

21 Fitzmaurice DA, Hobbs FD, Murray ET, Holder RL, Allan TF, Rose PE. Oral anticoagulation management in primary care with the use of computerized decision support and near-patient testing: a randomized, controlled trial. Arch Intern Med 2000;160:2343-2348. 